\title{
In patients with superficial vein thrombosis the inflammatory response is increased and related to the recanalization rate
}

\author{
Pavel Poredoš, Ana Spirkoska, Mateja Kaja Ježovnik
}

Department of Vascular Disease, University Medical Centre Ljubljana, Ljubljana, Slovenia

Submitted: 14 September 2016

Accepted: 14 March 2017

Arch Med Sci 2019; 15, 2: 393-401

DOI: https://doi.org/10.5114/aoms.2019.83292

Copyright @ 2019 Termedia \& Banach

\section{Abstract}

Introduction: Little is known about pathogenetic mechanisms of superficial venous thrombosis (SVT). We aimed to investigate the systemic inflammatory response in the acute phase of SVT, the time course of inflammatory markers and involvement of inflammation in resolution of thrombus in SVT. Material and methods: The circulatory inflammatory parameters high-sensitivity C-reactive protein (hsCRP), tumor necrosis factor $\alpha$ (TNF- $\alpha$ ), interleukins 6,8 and 10 (IL-6, IL-8, IL-10), and markers of fibrinolytic activity tissue plasminogen activator (t-PA), plasminogen activator inhibitor-1 (PAI-1) and fibrinogen were determined in 68 patients with acute SVT of lower limbs, who were allocated to two groups, dalteparin $5000 \mathrm{IU}$ once daily or $10000 \mathrm{IU}$ once daily. Recanalization of occluded veins was monitored by ultrasonography at regular intervals. Blood was drawn in the acute phase and after 12 weeks.

Results: In the acute phase a majority of the measured inflammatory markers were increased, while after 12 weeks most of them significantly dropped: hsCRP: $13.6 \pm 11.9$ vs. $7.4 \pm 4.4, p<0.001$; IL-6: $3.8 \pm 3.1$ vs. 2.6 $\pm 1.9, p=0.007$. Significant changes in endogenic fibrinolytic parameters were also observed: t-PA activity decreased $(0.81 \pm 0.35$ vs. $0.68 \pm 0.34$, $p=0.003)$, while PAl-1 levels increased $(5.6 \pm 5.1$ vs. $8.8 \pm 8.5, p<0.001)$. Levels of inflammatory markers at inclusion and after 12 weeks were related to less effective thrombus resolution: CRP: $r=0.386, p=0.001$; IL-6: $r=0.384$; TNF- $\alpha: r=0.255, p=0.037$.

Conclusions: In the acute phase of SVT, most of the circulating inflammatory markers were increased and most of their levels decreased after 12 weeks. Levels of inflammatory markers were negatively correlated with the recanalization rate.

Key words: superficial venous thrombosis, inflammation, anticoagulants, inflammatory markers, thrombophlebitis.

\section{Introduction}

Superficial vein thrombosis (SVT) is a multifactorial disease and similar risk factors to those in deep vein thrombosis are involved in its pathogenesis. In SVT varicose veins represent one the most important risk factors which predisposes to thrombus formation in superficial veins. Acquired or inherited risk factors potentially responsible for these disorders are identifiable in most patients. However, the basic pathogenetic

\author{
Corresponding author: \\ Prof. Pavel Poredoš PhD, MD \\ Department \\ of Vascular Disease \\ University Medical \\ Centre Ljubljana \\ 7 Zaloška St \\ 1000, Ljubljana, Slovenia \\ Phone: + 38641703038 \\ E-mail: pavel.poredos@kclj.si
}


mechanisms which stimulate inflammation and thrombus formation in superficial veins remain unexplained. The most recognizable pathophysiological mechanism through which risk factors cause SVT involve endothelial damage, blood stasis and changes in blood constituents [1, 2].

During the last decade, the role of inflammation in the etiopathogenesis of arterial thrombosis has been elucidated. However, less is known about the relationship between inflammation and venous thrombosis. Recently, inflammation has been accepted as a possible mechanism through which different risk factors trigger thrombus formation in veins. The data indicate that inflammation of the vessel wall initiates thrombus formation and that the inflammation and the coagulation system are coupled by common activation pathways [3]. Some studies have indicated that inflammation and hemostasis are also coupled by a feedback regulation system. During inflammation, the hemostatic balance may be disturbed by increased production of procoagulant factors and downregulation of anticoagulant mechanisms. Hence the activation of the coagulation cascade, with the formation of thrombin and fibrin deposition, could be the consequence of inflammation.

Inflammatory mechanisms also inhibit fibrinolytic activity and inflammatory mediators appear to increase the platelet activity. However, not only does inflammation lead to activation of coagulation, but coagulation also considerably affects inflammatory activity. Thus, thrombus formation promotes the inflammatory response, which stimulates the thrombotic tendency [4]. This thesis is supported by observations that natural anticoagulants not only prevent thrombosis but also suppress the inflammatory activity [5]. The crosslink between inflammation and hemostasis is complex and involves different reactions, endothelial damage and production of cell-derived micro-particles, which are released in the circulation [6].

As little is known about the relationship between inflammation and SVT, we aimed to investigate the systemic inflammatory response in patients with acute SVT, particularly the molecules that are most likely involved in the inflammation of the vessel wall (interleukins), to follow their levels up to three months after the initial thrombotic event and to elucidate whether systemic inflammatory response is related to the resolution of thrombi in the superficial veins.

\section{Material and methods}

\section{Study design and participants}

The study was a randomized, double-blind, single-center study carried out in an outpatient department of a tertiary academic institution. All eligible patients showing clinical signs of SVT referred from their primary care physicians were included in the study from December 2010 to December 2013.

The inclusion criteria were ultrasonographically confirmed first symptomatic presentation of an acute SVT of lower extremities ( $<5$ days after the onset of the symptoms, with an ultrasonographically confirmed thrombus length of at least $10 \mathrm{~cm}$ in the great saphenous vein, small saphenous vein, or major tributaries of the great saphenous vein, body weight 65 to $90 \mathrm{~kg}$, and age 18 to 85 years). Exclusion criteria included concomitant deep vein thrombosis (DVT) and/or pulmonary embolism, thrombosis extending closer than $5 \mathrm{~cm}$ to the sapheno-femoral junction or $3 \mathrm{~cm}$ to the sapheno-popliteal junction, inability to objectively confirm the diagnosis, history of previous venous thromboembolism (DVT, pulmonary embolism, or SVT), secondary vein thrombosis as a consequence of previous trauma or intravenous access, contraindications for anticoagulant treatment and thrombocytopenia $\left(<100 \times 10^{9} /\right.$ l). Also excluded were all patients with previous or recent malignancy, sepsis, autoimmune disorders, pregnancy, hormone therapy, hepatic or renal insufficiency (glomerular filtration rate $<50 \mathrm{ml} / \mathrm{min} / 1.73 \mathrm{~m}^{2}$ ), or receiving anti-inflammatory treatment (except aspirin $\leq 100 \mathrm{mg} /$ day). Throughout the study period, events such as recurrent SVT (in a previously recanalized section or on the contralateral leg), development of DVT or PE, and major hemorrhage were followed and considered as secondary end points.

The study was conducted according to the ethical principles stated in the Declaration of Helsinki. The study protocol was approved by the National Ethical Committee and all patients provided written consent. The study was registered at ClinicalTrails.gov under the number NCT01245998.

\section{Randomization of patients}

Patients were individually randomized by a computer-generated random allocation sequence. Randomization used block sizes of 4 . All participants and investigators were masked to treatment assignment and also blind to the size of each block.

Patients were randomly assigned to receive different dosages of dalteparin: the first group treated with preventive dosages of dalteparin $5000 \mathrm{IU}$ s.c. once daily and the second group treated with intermediate dosage of dalteparin 10000 IU s.c. once daily. Both groups received treatment with the study drug for 6 weeks; thereafter, only compression treatment was performed. From the beginning of the study all patients were advised to remain active and to use compression bandages or elastic compression stockings up to the groin (30-40 $\mathrm{mm} \mathrm{Hg}$ compression) for the whole study period. 


\section{Investigating procedures}

\section{Ultrasound of superficial veins in acute phase of SVT}

Before inclusion in the study, all patients underwent color-coded duplex ultrasonography and compression ultrasonography of all venous segments (from groin to ankle) of both lower limbs to confirm the diagnosis of SVT and to detect or exclude concomitant DVT. The location and extent of SVT (the proximal and distal end of the clot from the sapheno-femoral or sapheno-popliteal junction, the length of the thrombotic process measured using ultrasound and a measuring tape on the body surface and the diameter of the occluded superficial venous segment measured at 6 sites along the affected thrombosed vein) were determined. The diagnosis of SVT in the great saphenous vein, small saphenous vein, and/or their tributaries was established.

\section{Assessments and follow-up}

All data were collected prospectively. Patients attended follow-up visits at 6 weeks, 3 months, 6 months and 12 months. We recorded clinical details at baseline and at each visit. Body mass index (BMI) was calculated as weight (kg) divided by the square of the height $\left(\mathrm{m}^{2}\right)$. At each study visit besides complete clinical examination, ultrasound investigation of all deep and superficial veins on both lower extremities was also performed. At each check-up, the length of thrombus in the vein was measured and the recanalization rate was calculated as a percentage from the baseline length. The reduction in thrombus length was separately calculated in the proximal and the distal part, using the end of the thrombus as the baseline. Also the changes in maximal diameter of the occluded veins and the proximal and distal distance of the end of the thrombus to the sapheno-femoral or sapheno-popliteal junction were followed.

We gave every patient a mobile phone number of a study physician available $24 / 7$ to call if clinical manifestations suggestive of progression of SVT, deep vein thrombosis (swelling, tenderness or pain) or pulmonary embolism (sudden chest pain, new onset dyspnoea or tachycardia) occurred. Follow-up was stopped when ultrasonographic progression of thrombosis to the deep venous system, symptomatic DVT, or pulmonary embolism (confirmed by computed tomography) was observed. In patients who developed a thromboembolic event during follow-up, the study drug was discontinued and patients were admitted to hospital for further diagnostic and therapeutic anticoagulant treatment according to the recent guidelines for treatment of venous thromboembolism [7]. In addition, we instructed patients to re- port to the study physician on an emergency basis if any bleeding occurred.

\section{Laboratory methods}

Laboratory studies in all patients included complete blood cell count, kidney function tests, D-dimer assay, fibrinogen, markers of inflammation (high-sensitivity C-reactive protein - hsCRP, interleukin 6 - IL-6, interleukin $8-\mathrm{IL}-8$ and interleukin 10 - IL-10) and markers of endogenous fibrinolysis (tissue plasminogen activator - t-PA, and plasminogen activator inhibitor 1 - PAI-1). In all patients, blood samples were taken at the inclusion in the study (before administration of the study drug) and 3 months after the onset of SVT to ensure that the results were not affected by the thrombotic episode or anticoagulation therapy.

Fibrinogen concentration was determined according to the Clauss method, which is based on the determination of fibrinogen with thrombin clotting time, using commercially available reagents (Multifiber, Behring, German). D-dimer was determined by an immunoturbidimetric assay Auto-Dimer (Axis-Shield, Sweden) on an automated coagulation analyzer CS2100i (Sysmex, Japan). High-sensitivity C-reactive protein (hsCRP) was determined by an immunoturbidimetric assay (Vitros Fusion 5.1, Ortho-Clinical Diagnostics, USA). The concentrations of human tumor necrosis factor $\alpha$ (Quantikine HS High Sensitivity, Human TNF- $\alpha$ Immunoassay, R\&D Systems, USA), interleukin-6 (Quantikine HS High Sensitivity, Human IL-6 Immunoassay, R\&D Systems, USA), activity of plasminogen activator inhibitor-1 (PAI-1) (TriniLIZEPAI-1 Activity, AxisShield, Sweden) and plasminogen activator inhibitor-1 (PAl-1) antigen (TriniLIZEPAl-1 Antigen, Axis-Shield, Sweden) were measured by enzyme-linked immunosorbent assay (ELISA) using commercially available kits according to the manufacturers' instructions.

\section{Statistical analysis}

Categorical variables were presented as the ratio with the number of valid observations and continuous variables as average values. In basic comparative statistics, we applied the $\chi^{2}$ test and Fisher's exact test or related samples Wilcoxon signed rank test for categorical and continuous variables, respectively. A multivariate analysis was adjusted to all independent outcome predictors. To avoid variable selection caused by spurious correlations, only variable showing a relationship with the outcome (defined as $p<0.10$ in univariate model) were included as potential predictors. We identified the final multivariate model for each major outcome using a backward stepwise approach. A 2-tailed $p<0.05$ was considered sig- 
nificant. Statistical analyses were performed with SPSS for Windows, release 23.0 (IBM, USA).

\section{Results}

\section{Patients' characteristics and treatment}

A total of 119 consecutive patients were evaluated; 41 patients were not eligible due to previous venous thromboembolism, known cancer, concomitant disease or indications for indefinite anticoagulation, 6 patients refused to sign the informed consent, and 4 patients were not included because of high likelihood of being lost to follow-up (planned move out of state). Sixty-eight patients with first superficial venous thrombosis, included in the study, totally fulfilled the strict inclusion criteria: 33 patients received dalteparin $5000 \mathrm{IU}$ and 35 patients received dalteparin 10000 IU. There were non-significantly more females than males. Baseline clinical characteristics of patients were well balanced between groups (Table I). Most of the patients were overweight $(\mathrm{BMI}=29.9 \pm 5.1 \mathrm{~kg} /$ $\left.\mathrm{m}^{2}\right)$. The right leg was more frequently affected than the left leg $(67.5 \%$ vs. $32.5 \%, p=0.03)$ and only in $2(3 \%)$ patients were both legs affected. Superficial vein thrombosis was most frequently located in the main trunk of the great saphenous vein $(87 \%)$. In 52 (76\%) patients, varicose veins were registered; they were present in 25 (76\%) patients in the lower dosage group and in 27 (77\%) patients in the higher dosage group $(p=0.74)$. Fifty-nine $(87 \%)$ patients wore elastic compression stockings or elastic compression bandages all the time, 5 (8\%) occasionally (at least 3 days a week) and 3 (5\%) never. The compliance with treatment was high: all patients used the prescribed drug according to the instructions.

\section{Superficial venous thrombosis and inflammation}

In all patients the average length of thrombus was significantly reduced after 3 months $(p<0.01)$ and the average diameter of investigated veins at the end of the observation period was significantly smaller than at inclusion in the study ( $p<0.01$ ). Complete thrombus resolution and recanalization were observed in 23 patients after 3 months (34\%) and in 41 patients after 12 months (60\%). There were no significant differences between the group of patients treated with dalteparin $5000 \mathrm{IU}$ or $10000 \mathrm{IU}$.

At inclusion in the study most of the inflammatory markers were significantly increased; however, no significant differences in the levels of inflammatory markers were found between the treated groups. Also fibrinolytic parameters were comparable between the groups (Table II).

After 12 months of follow-up, a significant reduction of levels of inflammatory markers was observed, with the exception of IL-8 and anti-inflammatory IL-10, which did not significantly change in the observation period (Table III). There were no significant differences in changes of the blood levels of inflammatory markers between groups $A$ and $B$. It indicates that during the acute phase of SVT, there is an increased systemic inflammatory response and activation of endogenic fibrinolysis. However, with some exception, the levels of inflammatory markers declined after several weeks.

Table I. Clinical characteristics of patients with superficial vein thrombosis included in the study treated with dalteparin $10000 \mathrm{IU}$ (group A) and dalteparin $5000 \mathrm{IU}$ (group B) (values are given as mean \pm SD)

\begin{tabular}{|c|c|c|c|c|}
\hline Parameter & $\begin{array}{l}\text { Whole group } \\
\quad(n=68)\end{array}$ & $\begin{array}{l}\text { Group A } \\
(n=35)\end{array}$ & $\begin{array}{l}\text { Group B } \\
(n=33)\end{array}$ & $\begin{array}{l}P \text {-value } \\
\text { A vs. B }\end{array}$ \\
\hline Age [years] & $60.2 \pm 11.2$ & $59.4 \pm 11.7$ & $61.3 \pm 10.8$ & 0.42 \\
\hline Sex (male) & 31 (46\%) & $16(46 \%)$ & $15(46 \%)$ & 0.71 \\
\hline Height $[\mathrm{m}]$ & $1.68 \pm 0.09$ & $1.65 \pm 0.08$ & $1.68 \pm 0.10$ & 0.96 \\
\hline Weight [kg] & $83.7 \pm 15.0$ & $85.8 \pm 15.4$ & $79.9 \pm 16.0$ & 0.13 \\
\hline $\mathrm{BMI}\left[\mathrm{kg} / \mathrm{m}^{2}\right]$ & $29.9 \pm 5.1$ & $31.2 \pm 4.7$ & $28.6 \pm 5.3$ & 0.08 \\
\hline Systolic blood pressure [mm Hg] & $142.9 \pm 19.0$ & $141.1 \pm 18.9$ & $144.8 \pm 19.3$ & 0.43 \\
\hline Diastolic blood pressure [mm Hg] & $84.5 \pm 9.7$ & $84.0 \pm 9.8$ & $85.1 \pm 9.6$ & 0.64 \\
\hline Arterial hypertension & $20(29.4 \%)$ & $14(40.0 \%)$ & $6(18.2 \%)$ & 0.06 \\
\hline Diabetes mellitus & $1(1.5 \%)$ & $1(2.9 \%)$ & $0(0 \%)$ & 0.34 \\
\hline Hyperlipidemia & $12(17.6 \%)$ & $7(20.0 \%)$ & $5(15.2 \%)$ & 0.52 \\
\hline Smoking & $14(20.5 \%)$ & $6(17.1 \%)$ & $8(24.2 \%)$ & 0.10 \\
\hline Varicose veins & $52(76 \%)$ & $27(77 \%)$ & $25(76 \%)$ & 0.74 \\
\hline
\end{tabular}

$B M I$ - body mass index. 
The resolution of thrombosis appeared after 1 year and the length of thrombus was related to sex; males had longer thrombotic occlusions of affected veins in comparison to females (Table III); however, recanalization was negatively related to t-PA activity. The presence of classical risk factors of atherosclerosis did not influence the recanalization rate. Different drugs also had no significant influence on recanalization rate (Table IV).

Tables $V$ and $V I$ show that resolution of thrombus (reduction of its diameter and length) is significantly related to the levels of inflammatory markers, particularly interleukins, hsCRP and TNF- $\alpha$ in the acute phase and after 12 weeks.

Figure 1 shows the interrelationship between reduction of the length of thrombus and levels of hsCRP and IL-6.

\section{Discussion}

In one of our previous studies we showed that recanalization of occluded superficial veins is a long-lasting process, and the thrombus load (length and diameter of affected vein) was significantly reduced after 6 months of observation. However, after one year, complete recanalization was observed in less than $50 \%$ of patients [8]. It was also shown that in patients with increased thrombus load a longer period is needed for lysis of the thrombus. The results of this study also indicated that thrombus load and intrinsic fibrinolysis are probably more important for recanalization of occluded veins than the treatment regimen. In our study, one of the therapeutic options was chosen, in agreement with recent guidelines [9]. Recanalization rate and reduction in total thrombus

Table II. Values of laboratory investigations of inflammatory markers and fibrinolytic parameters at inclusion in the study in patients with superficial vein thrombosis treated with dalteparin $10000 \mathrm{IU}$ (group A) and dalteparin $5000 \mathrm{IU}$ (group B) (values are given as mean \pm SD)

\begin{tabular}{|c|c|c|c|}
\hline Parameter & Group A $(n=35)$ & Group B $(n=33)$ & $P$-value \\
\hline Fibrinogen [g/l] & $4.2 \pm 1.1$ & $3.8 \pm 1.2$ & 0.141 \\
\hline D-dimer [ng/ml] & $956 \pm 1019$ & $863 \pm 1215$ & 0.733 \\
\hline $\mathrm{HsCRP}[\mathrm{mg} / \mathrm{l}]$ & $13.2 \pm 10.2$ & $14.1 \pm 13.7$ & 0.745 \\
\hline IL-6 [ng/l] & $3.7 \pm 3.1$ & $3.9 \pm 3.2$ & 0.755 \\
\hline IL-8 [ng/l] & $19.3 \pm 13.8$ & $26.2 \pm 22.0$ & 0.125 \\
\hline IL-10 [ng/I] & $0.7 \pm 0.6$ & $0.5 \pm 0.5$ & 0.132 \\
\hline TNF- $\alpha[n g / l]$ & $2.6 \pm 6.4$ & $2.1 \pm 1.9$ & 0.680 \\
\hline t-PA [IU/ml] & $0.8 \pm 0.3$ & $0.8 \pm 0.3$ & 0.899 \\
\hline PAI-1 [IU/ml] & $6.3 \pm 5.4$ & $4.7 \pm 4.6$ & 0.182 \\
\hline
\end{tabular}

SD - standard deviation, hSCRP - high-sensitivity C-reactive protein, IL-6 - interleukin 6, IL-8 - interleukin 8, IL-10 - interleukin 10, TNF- $\alpha$-tumor necrosis factor $\alpha, t$-PA - tissue plasminogen activator, PAl-1 - plasminogen activator inhibitor-1.

Table III. Values of laboratory investigations of inflammatory markers and fibrinolytic parameters of both groups of patients with superficial vein thrombosis at inclusion in the study (acute phase of the disease) and after 12 weeks of follow-up (values are given as mean \pm SD)

\begin{tabular}{|c|c|c|c|}
\hline Parameter & At inclusion $(n=68)$ & After 12 weeks $(n=68)$ & $P$-value \\
\hline Fibrinogen $[\mathrm{g} / \mathrm{l}]$ & $4.1 \pm 1.3$ & $3.6 \pm 0.7$ & 0.03 \\
\hline D-dimer [ng/ml] & $1019.28 \pm 127$ & $428 \pm 69$ & 0.007 \\
\hline $\mathrm{HsCRP}[\mathrm{mg} / \mathrm{l}]$ & $13.61 \pm 11.89$ & $7.35 \pm 4.41$ & $<0.001$ \\
\hline IL-6 [ng/l] & $3.81 \pm 3.11$ & $2.62 \pm 1.88$ & 0.007 \\
\hline IL-8 [ng/l] & $22.39 \pm 18.14$ & $21.91 \pm 18.76$ & 0.634 \\
\hline IL-10 [ng/l] & $0.63 \pm 0.57$ & $0.62 \pm 0.58$ & 0.863 \\
\hline TNF- $\alpha[n g / I]$ & $2.37 \pm 4.96$ & $1.46 \pm 0.82$ & 0.129 \\
\hline $\mathrm{t}-\mathrm{PA}[\mathrm{IU} / \mathrm{ml}]$ & $0.81 \pm 0.35$ & $0.68 \pm 0.34$ & 0.003 \\
\hline PAI-1 [IU/ml] & $5.58 \pm 5.13$ & $8.77 \pm 8.47$ & $<0.001$ \\
\hline
\end{tabular}

SD - standard deviation, HSCRP - high-sensitivity C-reactive protein, IL-6 - interleukin 6, IL-8 - interleukin 8, IL-10 - interleukin 10, TNF- $\alpha$-tumor necrosis factor $\alpha, t$-PA - tissue plasminogen activator, PAl-1 - plasminogen activator inhibitor-1. 
load were not significantly different between the groups of patients treated with 5000 IU or 10000 IU of dalteparin.

Thrombus resolution of occluded veins was faster in women than in men. Males and females were comparable in age and thrombus load; there-

Table IV. Influence of risk factors of atherosclerosis and drug treatment on resolution of thrombus (reduction of length between first (acute phase) and last (after 1 year) examination) ( $r$ - Pearson correlation coefficient)

\begin{tabular}{|lll|}
\hline Parameter & $r$ & $P$-value \\
\hline Risk factors: & \\
\hline Sex (F/M) & 0.414 & $<0.001$ \\
\hline Age & 0.080 & 0.518 \\
\hline BMI & 0.160 & 0.191 \\
\hline AH & 0.090 & 0.467 \\
\hline HLP & 0.026 & 0.836 \\
\hline DM & 0.188 & 0.124 \\
\hline Smoking & -0.032 & 0.799 \\
\hline Drug treatment: & & \\
\hline ACE inhibitors & 0.090 & 0.467 \\
\hline Statins & 0.026 & 0.836 \\
\hline Antidiabetics & 0.188 & 0.124 \\
\hline Aspirin & 0.212 & 0.087 \\
\hline NSAID & 0.040 & 0.745 \\
\hline
\end{tabular}

$B M I$ - body mass index, $A H$ - arterial hypertension, HLP - hyper lipidemia, DM - diabetes mellitus, NSAID - nonsteroidal antiinflammatory drugs. fore other factors such as differences in hormonal status probably influenced faster resolution of thrombus in females.

In the process of thrombus resolution, the inflammation that influences endogenic fibrinolytic potential probably plays an important role in recanalization of superficial and deep venous thrombosis.

It seems that during a comparable observation period, recanalization of SVT happens less frequently than in deep veins [10]. Recanalization of occluded veins is characterized by the interplay of factors which favor formation of a new thrombus and those that tend to degrade venous thrombus and restore the venous lumen and venous flow. In our previous study we also showed that resolution of thrombus in deep veins is faster in women in comparison to men. In our previous study we found increased levels of circulating inflammatory markers in patients with idiopathic DVT, which were related to endothelial dysfunction [11]. However, there are no data on the role of inflammatory markers in the etiopathogenesis of SVT, but they probably act through similar mechanisms as in DVT.

In our study, in the acute phase of SVT a majority of inflammatory markers were increased, particularly hsCRP, IL- 6 and TNF- $\alpha$. This finding was expected, since inflammation represents one of the basic pathogenetic mechanisms of SVT and is not limited only to the vessel wall, but usually affects the surrounding tissue as well. However, some inflammatory markers, particularly cytokines, are probably specific indicators of inflammation of the vessel wall. Circulating cytokines interact with specific receptors on various cells including endothelial cells; this leads to the inflammatory re-

Table V. Influence of levels of systemic inflammatory markers (CRP, IL-6, IL-8, IL-10, TNF- $\alpha$ ) and fibrinolytic parameters (t-PA and PAI-1) on thrombus diameter in acute phase and after 1 year in patients with superficial vein thrombosis Blood samples were taken in the acute phase of the disease and after 12 weeks ( $r$-Pearson correlation coefficient)

\begin{tabular}{|c|c|c|c|c|}
\hline \multirow[t]{2}{*}{ Parameter } & \multicolumn{2}{|c|}{ Acute phase of the disease } & \multicolumn{2}{|c|}{ After 1 year } \\
\hline & $r$ & $P$-value & $r$ & $P$-value \\
\hline \multicolumn{5}{|c|}{ Systemic inflammatory markers: } \\
\hline HsCRP & 0.153 & 0.210 & 0.386 & 0.001 \\
\hline IL-6 & 0.357 & 0.003 & 0.384 & 0.001 \\
\hline IL-8 & 0.080 & 0.518 & 0.112 & 0.368 \\
\hline IL-10 & 0.106 & 0.393 & 0.230 & 0.061 \\
\hline TNF- $\alpha$ & 0.047 & 0.705 & 0.255 & 0.037 \\
\hline \multicolumn{5}{|c|}{ Systemic fibrinolytic parameters: } \\
\hline$t-P A$ & 0.245 & 0.050 & 0.041 & 0.746 \\
\hline PAI-1 & -0.195 & 0.114 & -0.028 & 0.825 \\
\hline
\end{tabular}

CRP - C-reactive protein, IL-6-interleukin 6, IL-8 - interleukin 8, IL-10-interleukin 10, TNF- $\alpha$ - tumor necrosis factor $\alpha, t$-PA - tissue plasminogen activator, PAl-1 - plasminogen activator inhibitor-1. 
Table VI. Influence of levels of systemic inflammatory markers (CRP, IL-6, IL-8, IL-10, TNF- $\alpha$ ) and fibrinolytic parameters (t-PA and PAI-1) on thrombus length at inclusion and after 1 year in patients with superficial vein thrombosis ( $r$ - Pearson correlation coefficient)

\begin{tabular}{|c|c|c|c|c|}
\hline \multirow[t]{2}{*}{ Parameter } & \multicolumn{2}{|c|}{ Thrombus length at inclusion } & \multicolumn{2}{|c|}{ Thrombus length after 1 year } \\
\hline & $r$ & $P$-value & $r$ & $P$-value \\
\hline \multicolumn{5}{|c|}{ Systemic inflammatory markers: } \\
\hline CRP & 0.167 & 0.175 & 0.256 & 0.035 \\
\hline IL-6 & 0.058 & 0.640 & 0.331 & 0.006 \\
\hline IL-8 & 0.082 & 0.507 & 0.040 & 0.745 \\
\hline IL-10 & 0.202 & 0.101 & 0.214 & 0.082 \\
\hline TNF- $\alpha$ & 0.162 & 0.192 & 0.256 & 0.036 \\
\hline \multicolumn{5}{|c|}{ Systemic fibrinolytic parameters: } \\
\hline $\mathrm{t}-\mathrm{PA}$ & 0.004 & 0.975 & 0.096 & 0.445 \\
\hline PAl-1 & 0.062 & 0.615 & -0.069 & 0.580 \\
\hline
\end{tabular}

CRP - C-reactive protein, IL-6 - interleukin 6, IL-8-interleukin 8, IL-10 - interleukin 10, TNF- $\alpha$ - tumor necrosis factor $\alpha$, t-PA - tissue plasminogen activator, PAl-1 - plasminogen activator inhibitor-1.

A

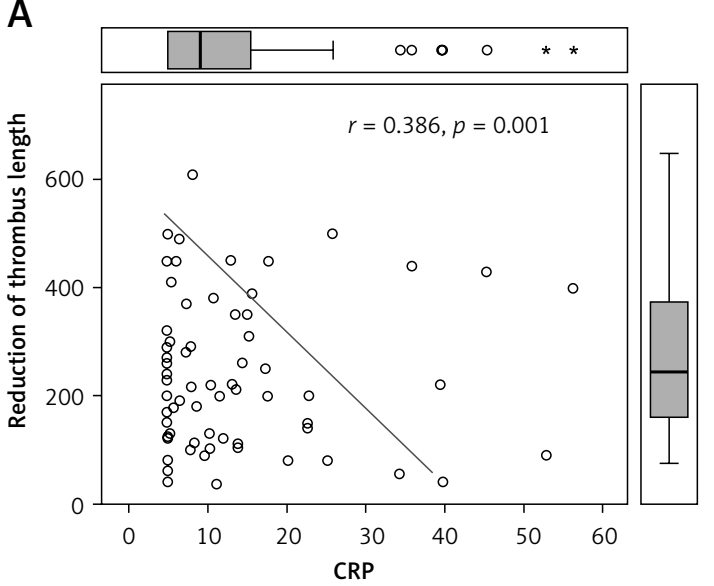

B

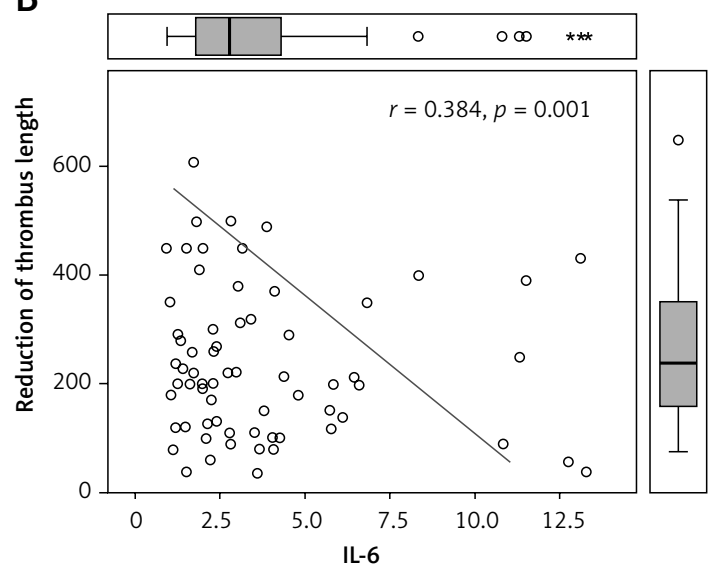

Figure 1. Relationship between systemic inflammatory markers (high-sensitivity C-reactive protein, interleukin 6) and recanalization rate (reduction of thrombus length) of thrombosed superficial veins

sponse, involving blood cell adhesion to endothelial cells, increased permeability of endothelium and apoptosis [12]. Increased levels of cytokines are associated with vascular diseases such as varicose veins and atherosclerosis. Pro-inflammatory cytokines are known to stimulate production of reactive oxygen species in endothelial cells of blood vessels and to decrease bioavailability of nitrogen oxide [13]. On the other hand, anti-inflammatory IL-10 provides some protection against endothelial dysfunction and diminishes the inflammatory response [14]. Damage of the vessel wall caused by inflammation is probably a crucial event in the initiation of thrombus formation. Different studies have indicated that inflammation is a possible mechanism through which different risk factors trigger thrombus formation in veins [15]. During inflammation, there is increased production of pro-coagulant factors [16]. Inflammation can also trigger thrombus formation through deterioration of venous wall function. Inflammation increases the oxidative stress with decreased bio-availability of nitrogen oxide and consequently endothelial mediated relaxation and functional capability of blood vessels are deteriorated [17]. Inflammation also promotes coagulation through its inhibitory effect on endogenic fibrinolytic activity and morphological deterioration of the vessel wall. High-sensitivity CRP promotes the prothrombotic state through induction of synthesis of tissue factor and activation of the extrinsic coagulation pathway.

Cytokines are probably directly involved in thrombogenesis and are not merely a consequence of inflammation in the acute phase of the disease. Cytokines may also have prognostic value in the development of first and recurrent thrombosis. In the Leiden Thrombophilia Study (LETS), 
cytokines IL-6, IL-8 and TNF- $\alpha$ were associated with 2-3 fold increased risk for first DVT [18].

In our study, 12 weeks after the acute episode of SVT a significant decrease of some inflammatory markers (hsCRP, IL-6) was observed. Significant changes in fibrinolytic parameters were also observed (t-PA decreased and PAI -1 increased). The reduction of levels of inflammatory and pro-fibrinolytic markers after the acute phase of the disease indicates that these changes are transitory and are mostly the consequence and not the primary cause of the disease. The primary cause of SVT is probably the damaged vessel wall. As SVT usually develops in varicose veins, turbulent blood flow in varicose convolutes damages the vessel wall, which triggers the inflammatory response [19].

One of the proposed mechanisms triggering thrombus formation is intravascular tissue factor, which initiates coagulation via circulating micro-vesicles and platelets. This enables the entire coagulation system to proceed on a restricted cell surface [5]. Levels of extracellular vesicles were found to be increased in inflammation [20], which indicates that inflammation also promotes coagulation through formation of extracellular vesicles. Furthermore, the results of some studies indicated that heparin has anti-inflammatory and anticoagulant effects due to suppression of extracellular vesicles released from the cells [21]. However, in our study the effect of dalteparin on extracellular microvesicles was not followed, but the recanalization rate between the groups of patients receiving 5000 or $10000 \mathrm{IU}$ dalteparin was comparable. This may indicate that a saturation effect was reached with a low dosage of dalteparin and higher dosage had no additional anticoagulant or thrombolytic effect.

We were the first to show that recanalization of thrombotic occlusion of superficial veins is related to levels of inflammatory markers in the acute phase of the disease and after 12 weeks of follow-up. The resolution of thrombi in superficial veins is a long-lasting process and complete recanalization occurs only in about half of patients, as shown in our previous study [8].

The levels of interleukins, CPR and TNF- $\alpha$ in the acute phase of the disease and after 12 weeks were significantly related to a lower recanalization rate of occluded superficial veins. These results indicate pro-coagulant activity of the inflammatory process and its inhibition of endogenic fibrinolysis. Similarly, also in the study of recanalization in DVT, increased cytokine levels were related to less effective thrombus resolution [10].

These results indicate that determination of some inflammatory markers may help in prognostication of outcome of SVT. However, the investigated markers are non-specific and their reproducibility is limited. Therefore, their use in everyday practice is not appropriate.

In conclusion, in patients with SVT, levels of inflammatory markers are increased in the acute phase of the disease. Increased levels of interleukins may indicate inflammation of the vessel wall. Most of the markers significantly decrease after 12 weeks.

Levels of circulating inflammatory markers are negatively related to the recanalization rate of thrombosed superficial veins, which indicates that inflammation due to its procoagulant and antifibrinolytic effects inhibits the resolution of thrombi and the recanalization rate of occluded veins.

\section{Acknowledgments}

The study was conducted with the support of the research program P3-0308 financed by the Slovenian Research Agency.

\section{Conflict of interest}

The authors declare no conflict of interest.

\section{References}

1. Prandoni P, ten Cate JW. Epidemiology, risk factors, and natural history of venous thromboembolism (I). Pulmonary embolism. Blackwell Science, Berlin 1999; 2-16.

2. Decousus H, Quéré I, Presles E, et al.; POST (Prospective Observational Superficial Thrombophlebitis) Study Group. Superficial venous thrombosis and venous thromboembolism: a large, prospective epidemiologic study. Ann Intern Med 2010; 152: 218-24.

3. Aksu K, Donmez A, Keser G. Inflammation-induced thrombosis: mechanisms, disease associations and management. Curr Pharm Des 2012; 18: 1478-93.

4. Collen D, Hoylaerts MF. Relationship between inflammation and venous thromboembolism as studied by microparticle assessment in plasma. J Am Coll Cardiol 2005; 45: 1472-3.

5. Müller I, Klocke A, Alex M, et al. Intravascular tissue factor initiates coagulation via circulating microvesicles and platelets. FASEB J 2003; 17: 476-8.

6. Esmon CT. Inflammation and thrombosis. J Thromb Haemost 2003; 1: 1343-8.

7. Kearon C, Akl EA, Comerota AJ, et al. Antithrombotic therapy for VTE disease: antithrombotic therapy and prevention of thrombosis, $9^{\text {th }}$ ed: American College of Chest Physicians Evidence-Based Clinical Practice Guidelines. Chest 2012; 141: e419S-96S.

8. Spirkoska A, Jezovnik MK, Poredos P. Time course and the recanalization rate of superficial vein thrombosis treated with low-molecular-weight heparin. Angiology 2015; 66: 381-6.

9. Kalodiki E, Stvrtinova V, Allegra C, et al. Superficial vein thrombosis: a consensus statement. Int Angiol 2012; 31: 203-16.

10. Jezovnik MK, Poredos P. Factors influencing the recanalisation rate of deep venous thrombosis. Int Angiol 2012; 31: 169-75.

11. Jezovnik MK, Poredos P, Stalc M. Impairment of the vasodilatation capability of the brachial artery in pa- 
tients with idiopathic venous thrombosis. J Atheroscler Thromb 2010; 17: 1190-8.

12. Sprague AH, Khalil RA. Inflammatory cytokines in vascular dysfunction and vascular disease. Biochem Pharmacol 2009; 78: 539-52.

13. Rahman A, Kefer J, Bando M, Niles WD, Malik AB. E-selectin expression in human endothelial cells by TNF-alpha-induced oxidant generation and NF-kappaB activation. Am J Physiol 1998; 275: L533-44.

14. Poredos P, Jezovnik MK. In patients with idiopathic venous thrombosis, interleukin-10 is decreased and related to endothelial dysfunction. Heart Vessels 2011; 26: 596-602.

15. Wakefield TW, Strieter RM, Prince MR, Downing LJ, Greenfield LJ. Pathogenesis of venous thrombosis: a new insight. Cardiovasc Surg 1997; 5: 6-15.

16. Conde I, López JA. Classification of venous thromboembolism (VTE). Role of acute inflammatory stress in venous thromboembolism. J Thromb Haemost 2005; 3: 2573-5.

17. Clapp BR, Hingorani AD, Kharbanda RK, et al. Inflammation-induced endothelial dysfunction involves reduced nitric oxide bioavailability and increased oxidant stress. Cardiovasc Res 2004; 64: 172-8.

18. Reitsma PH, Branger J, Van Den Blink B, Weijer S, Van Der Poll T, Meijers JCM. Procoagulant protein levels are differentially increased during human endotoxemia. J Thromb Haemost 2003; 1: 1019-23.

19. Poredos P, Spirkoska A, Rucigaj T, et al. Do blood constituents in varicose veins differ from the systemic blood constituents? Eur J Vasc Endovasc Surg 2015; 50: 250-6.

20. Distler JHW, Pisetsky DS, Huber LC, Kalden JR, Gay S, Distler O. Microparticles as regulators of inflammation: novel players of cellular crosstalk in the rheumatic diseases. Arthritis Rheum 2005; 52: 3337-48.

21. Sustar V, Jansa R, Frank M, et al. Suppression of membrane microvesiculation: a possible anticoagulant and anti-tumor progression effect of heparin. Blood Cells Mol Dis 2009; 42: 223-7. 